Max-Planck-Institut für demografische Forschung

Max Planck Institute for Demographic Research

Konrad-Zuse-Strasse $1 \cdot$ D-18057 Rostock - GERMANY

Tel +49 (0) 3812081 - 0; Fax +49 (0) 3812081 - 202;

http://www.demogr.mpg.de

MPIDR WORKING PAPER WP 2005-024

AUGUST 2005

\title{
Shocks in homogeneous and heterogeneous populations
}

Maxim Finkelstein (FinkelM.SCI@mail.uovs.ac.za)

This working paper has been approved for release by: James W. Vaupel (jwv@ demogr.mpg.de) Head of the Laboratory of Survival and Longevity.

(C) Copyright is held by the authors.

Working papers of the Max Planck Institute for Demographic Research receive only limited review. Views or opinions expressed in working papers are attributable to the authors and do not necessarily reflect those of the Institute. 


\title{
SHOCKS IN HOMOGENEOUS AND HETEROGENEOUS POPULATIONS
}

\author{
Maxim Finkelstein \\ Department of Mathematical Statistics \\ University of the Free State \\ PO Box 339, 9300 Bloemfontein, Republic of South Africa \\ (e-mail: FinkelM@sci.uovs.ac.za) \\ and \\ Max Planck Institute for Demographic Research, \\ Rostock, Germany
}

\begin{abstract}
A system subject to a point process of shocks is considered. Shocks occur in accordance with a nonhomogeneous Poisson process. Different criterions of system failures are discussed in a homogeneous case. Two natural settings are analyzed. Heterogeneity is modeled by an unobserved univariate random variable (frailty). It is shown that reliability (safety) analysis for a heterogeneous case can differ dramatically from that for a homogeneous setting. A shock burn-in procedure for a heterogeneous population is described. The corresponding bounds for the failure rates are obtained.
\end{abstract}

Keywords: homogeneous populations, heterogeneous populations, frailty, shocks, burn-in, mixture failure rate.

\section{INTRODUCTION}

Consider a general orderly (without multiple occurrences) point process $\left\{T_{n}\right\} ; T_{0}=0, T_{n+1}>T_{n}, n=0,1,2, \ldots$, where $T_{n}$ is time to the $n$th arrival of an event with the corresponding cumulative distribution function $(\mathrm{Cdf}) F^{(n)}(t)$. An event at $t=0$ is only formal, denoting the starting point of a process. Let $G$ be a geometric variable 
with parameter $\theta$ (independent of $\left.\left\{T_{n}\right\}_{n \geq 0}\right)$ and $W(t, \theta)$ denote the corresponding geometric sum of lifetimes:

$$
W(t, \theta)=\theta \sum_{k=1}^{\infty} \bar{\theta}^{k-1} F^{(k)}(t),
$$

A natural reliability (or safety) interpretation of model (1) is via the stochastic point process of shocks. Let $T$ denote a random time to failure of a system subject to a point process of shocks. We interpret the word shock in a very broad sense as some instantaneous, potentially harmful event. Assume for simplicity that a shock is the only cause of a system failure. It means that the system is 'absolutely reliable' in the absence of shocks. The corresponding generalization on the case of non-reliable system as such can be easily performed under a natural assumption of independence between $\left\{T_{n}\right\}_{n \geq 0}, G$ and the system lifetime random variable $T$. Dealing with dependency presents substantial mathematical problems and should be considered as a special topic.

Assume also that each shock (except the shock at $t=0$, which is harmless and indicates, as was mentioned, the starting point of a process) independently of the previous history leads to a system failure with probability $\theta$ and is survived with probability $\bar{\theta}$. This procedure defines a terminating point process, and a system survival probability (reliability) in $(0, t)$ is

$$
S(t, \theta) \equiv 1-W(t, \theta)
$$

Thus all random shocks to occur in $(0, t)$ will be survived with probability $S(t, \theta)$.

Various shock models in reliability and safety analysis interpretation were considered by Barlow and Proschan [1], Thompson [2], Aven and Jensen [3], and Finkelstein [4] to name a few. All of them consider special cases of a point process. In the case of a renewal process, the inter-arrival times are i.i.d. random variables with an absolutely continuous common Cdf

$$
F(t)=1-\exp \left\{-\int_{0}^{t} \lambda(u) d u\right\},
$$

where $\lambda(t)$ is the corresponding failure rate. Therefore, the survival probability can be written as

$$
S(t, \theta) \equiv 1-W(t, \theta)=(1-F(t))+\sum_{k=1}^{\infty} \bar{\theta}^{k} F^{(k)}(t)
$$


where $F^{(k)}(t)$ is a $k$-fold convolution of $F(t)$ with itself and $F^{(0)}(t) \equiv F(t)$.

Special complicated numerical methods should be used for obtaining $S(t, \theta)$ in the form of the infinite series (3). Hence, it is very important for practical assessment of safety or reliability to obtain simple approximations and bounds. It is well known (see e.g. Kalashnikov [5]) that, as $\theta \rightarrow 0$, the following convergence in distribution takes place for the terminating renewal process:

$$
S(t, \theta) \rightarrow \exp \left\{-\frac{\theta t}{\mu}\right\}, \quad \forall t \in(0, \infty),
$$

where the mean $\mu$ of the $\operatorname{Cdf} F(t)$ is assumed to exist.

Specifically, when all inter-arrival times are exponentially distributed random variables with constant failure rate $\lambda,(4)$ turns into identity:

$$
S(t, \theta)=\exp \{-\theta \lambda t\} \text {. }
$$

Thus, relation (4) gives a very simple asymptotic exponential approximation. In practical situations in reliability, safety and risk analysis, however, parameter $\theta$ is not usually sufficiently small and therefore relation (4) can be used only like a very rough estimate.

\section{POISSON PROCESS OF SHOCKS}

The situation is much simpler from a computational point of view if the process of shocks is the Poisson one. At many instances this case is more realistic in practical modeling than the renewal one, as the nonhomogeneous Poisson process of shocks can model a natural dependence of intensity of shocks on time. Note, that the intensity of shocks for the renewal process (renewal density function) is a complex function, which tends to a constant, as $t \rightarrow \infty$. Therefore, the Poisson model of external influences is usually more adequate in practice than a renewal one.

For the Poisson process of shocks relation (3) is formally the same:

$$
S(t, \theta)=(1-F(t))+\sum_{k=1}^{\infty} \bar{\theta}^{k} F^{(k)}(t),
$$

but $F^{(k)}(t)$ denotes in this case the Cdf of time to the $k$-th shock arrival in the Poisson process of shocks with intensity $\lambda(t)$, which is a well-known distribution. Spe- 
cifically, the distribution of time to the first arrival is defined by relation (2). Our interest in what follows will be mostly in the Poisson process of shocks.

It turns out that in this case $S(t, \theta)$ can be obtained exactly in a very simple, speaking for itself form even for the time-dependent probability of termination $\theta(t)$, where $t$ is the chronological time since the start of the shock process. Let $\lambda\left(t, H_{t}\right)$ denote the complete intensity function for some general orderly point process (Cox and Isham [6]), where $H_{t}$ is a history of the process up to $t$ (the concrete configuration of arrival points in $[0, t))$. The value $\lambda\left(t, H_{t}\right) d t$ can be interpreted as the probability of an event occurrence in $[t, t+d t)$ for the given history $H_{t}$ in $[0, t)$. An important feature of this function is that unlike ordinary intensity $\lambda(t)$, the complete intensity 'completely' defines a point process [6]. It is clear that the conditional rate of termination $\lambda_{c}\left(t, H_{t}\right)$ for the general point process of shocks can be defined via the following equation

$$
\lambda_{c}\left(t, H_{t}\right) d t=\operatorname{Pr}\left\{T \in[t, t+d t) \mid H_{t}, T\left(H_{t}\right) \geq t\right\}=\theta(t) \lambda\left(t, H_{t}\right) d t .
$$

Condition $T\left(H_{t}\right) \geq t$ means that all shocks in $[0, t)$ were survived (for the specific configuration of shocks given by the history $H_{t}$ ). Conditional rate of termination is based on the internal 'individual' information (Kalbfleisch and Prentice [7]) and bears no usual exponential relationship with the corresponding survival function in a general case. At the same time, as $\lambda\left(t, H_{t}\right)=\lambda(t)$ for the specific case of the Poisson process of shocks relation (7) turns into:

$$
\lambda_{c}\left(t, H_{t}\right)=\theta(t) \lambda(t)
$$

and, finally the survival probability is

$$
S(t, \theta)=\exp \left\{-\int_{0}^{t} \theta(u) \lambda(u) d u\right\} .
$$

The foregoing can be considered as a "non-technical" proof of (9). The formal proof for the different setting can be found, for instance, in Block et al [8]. This result is very simple and we shall apply it in Section 4 to a heterogeneous case.

\section{WEAKER CRITERION OF FAILURE}


In the previous section the system could be killed by a shock and it was assumed to be 'as good as old', if a shock was survived (the analogue of a minimal repair). Assume that we are looking now at the process of non-killing shocks, but a failure of a system can still occur when the shocks are 'too close' and the system did not recover from the consequences of a previous shock. Therefore, the time for recovering should be taken into account. It is natural to assume that it is a random variable $\tau$ with a $\operatorname{Cdf} R(t)$ (different values of damage need different time of recovering and this fact is described by $R(t))$. Thus, if the shock occurs while the system still did not recover from the previous one, then a failure occurs. It is the simplest criterion of failure of this kind. Other criterions can be also considered. As previously, we want to derive the probability of a failure-free performance in $[0, t): S(t)$. Consider the Poisson process of shocks with rate $\lambda(t)$. Similar to Finkelstein and Zarudnij [9], the following integral equation for $S(t)$ can be derived:

$$
\begin{aligned}
& S(t)=\exp \left\{-\int_{0}^{t} \lambda(u) d u\right\}\left(1+\int_{0}^{t} \lambda(u) d u\right) \\
& +\int_{0}^{t} \lambda(x) \exp \left\{-\int_{0}^{x} \lambda(u) d u\right\}\left[\int_{0}^{t-x} \lambda(y) \exp \left\{-\int_{0}^{y} \lambda(u) d u\right\} R(y) \tilde{S}(t-x-y) d y\right] d x,
\end{aligned}
$$

where the first term in the right hand side is the probability that there was not more than one shock in $[0, t)$ and the integrand defines the joint probability of the following events

-the first shock occurred in $[x, x+d x)$,

-the second shock occurred in $[x+y, x+y+d y)$,

-the time between two shocks $y$ is sufficient for recovering (probability- $R(y)$ ),

-the system is functioning without failures in $[x+y, t)$.

By $\tilde{S}(t)$ in (10) we denote the probability of system's functioning without failures in $[0, t)$ given that the first shock had occurred at $t=0$. Thus, it differs from $S(t)$ by the initial state of a shock process. Note, that in contrast to previous sections, it is important now that in the initial setting there is no shock at $t=0$. Similar to (10): 


$$
\tilde{S}(t)=\exp \left\{-\int_{0}^{t} \lambda(u) d u\right\}+\int_{0}^{t} \lambda(x) \exp \left\{-\int_{0}^{x} \lambda(u) d u\right\} R(x) \tilde{S}(t-x) d x
$$

Equations (10) and (11) can be solved numerically. On the other hand, for the constant failure rate $\lambda$ these equations can be easily solved via the Laplace transform. Obtaining the Laplace transform of $\tilde{S}(t)$ from (11), and using this result while dealing with equation (10), we finally arrive at:

$$
S(s)=\frac{s[1-\lambda \tilde{R}(s+\lambda)]-\lambda^{2} R(s+\lambda)+2 \lambda}{(s+\lambda)^{2}[1-\lambda R(s+\lambda)]},
$$

where $S(s)$ and $R(s)$ denote Laplace transforms of $S(t)$ and $R(t)$, respectively. Consider important for practice specific cases:

Example 1. Exponentially distributed $\tau: R(t)=1-\exp \{-\mu t\}$. Then:

$$
R(s+\lambda)=\frac{\mu}{(s+\lambda)(s+\lambda+\mu)}
$$

and

$$
S(s)=\frac{s+2 \lambda+\mu}{s^{2}+s(2 \lambda+\mu)+\lambda^{2}}
$$

Performing the inverse Laplace transform:

$$
S(t)=A_{1} \exp \left\{s_{1} t\right\}+A_{2} \exp \left\{s_{2} t\right\}
$$

where $s_{1}, s_{2}$ are the roots of the denominator in (13):

$$
s_{1,2}=\frac{-(2 \lambda+\mu) \pm \sqrt{(2 \lambda+\mu)^{2}-4 \lambda^{2}}}{2}
$$

and

$$
A_{1}=\frac{s_{1}+2 \lambda+\mu}{s_{1}-s_{2}}, A_{2}=-\frac{s_{2}+2 \lambda+\mu}{s_{1}-s_{2}}
$$

Equation (14) gives an exact solution for $S(t)$. In applications it is convenient to use simple approximate formulas. Consider the following reasonable assumption 


$$
\frac{1}{\lambda}>>\bar{\tau} \equiv \int_{0}^{\infty}(1-R(x)) d x
$$

Relation (15) means that the mean inter-arrival time in the shock process is much larger than the mean time of recovery, and this is often the case in practice. In the study of repairable systems the similar case is usually called the fast repair. Therefore, using this assumption:

$$
S(t) \approx \exp \left\{-\lambda^{2} \bar{\tau} t\right\}
$$

as the second term in the right hand side of (14) decreases very sharply with $t$ $(\sim \exp \{-\bar{\tau} t\})$.

\section{GENERAL HETEROGENEOUS SETTING:}

One can hardly find homogeneous populations in real life, although most of reliability studies deal with a homogeneous case. Neglecting existing heterogeneity can lead to substantial errors in stochastic analysis in reliability, survival and risk analysis and other disciplines.

The simplest and the most natural way to implement heterogeneity in a model, is via a subjective approach, which deals with a random (unobserved) parameter and we shall follow this concept in the current paper. This parameter is often called "frailty" (Vaupel et al [10]). As an example of this approach we shall generalize a shock model of Section 2 to the heterogeneous case. Let probability $\theta(t)=\theta$ be constant in time for simplicity and assume that it is a random variable (independent of a shock process) with support in $[0,1]$. By this, in fact, we mean that $\theta$ is indexed by some random parameter. The case of a corresponding stochastic process $\theta_{t}, t \geq 0$ can be also considered. Then the setting can be interpreted in a following way: there is a population of objects (systems), which differ with respect to unobserved susceptibility to shocks. Therefore, we can divide our population into different homogeneous in susceptibility to shocks subpopulations and describe this operation by an unobserved random variable.

It follows from Yashin and Manton [11] and Finkelstein and Esaulova [12] that, as relations (8) and (9) are valid conditionally on realizations of $\theta$, the following formulas take place, when $\theta$ is a random variable: 


$$
\begin{gathered}
S_{m}(t)=E\left[\exp \left\{-\theta \int_{0}^{t} \lambda(u) d u\right\}\right] \\
\lambda_{m}(t)=\lambda(t) E[\theta \mid T \geq t]
\end{gathered}
$$

where conditional expectations are obtained with respect to $\theta$. Specifically, expectation in relation (17) defines the expected value of $\theta$ on condition that a system did not fail in $[0, t]$. Equations (16) and (17) define, the mixture (observed) survival function $S_{m}(t)$ and the mixture (observed) failure rate $\lambda_{m}(t)$, respectively. The 'shape' of $\lambda_{m}(t)$ in (17) can be already dramatically different from the shape of the intensity of the Poisson process $\lambda(t)$, whereas it is the same for the case of a constant in time $\theta(t) \equiv \theta$ in (8).

Another and maybe much more important from the practical point of view source of heterogeneity in harmful events can be modeled by the doubly stochastic Poisson process $\hat{P}_{t}, t \geq 0$ (instead of the Poisson process of harmful events). Denote a random rate of this process by $\lambda(t, \Psi)[6]$, where $\Psi$ is a random variable with support in $[0, \infty)$. For a fixed $\Psi=\psi$, similar to relations (8)-(9):

$$
\begin{gathered}
S(t \mid \psi)=\exp \left\{-\int_{0}^{t} \theta(u) \lambda(u, \psi) d u\right\}, \\
\lambda_{\psi}(t)=\theta(t) \lambda(t, \psi)
\end{gathered}
$$

and, similar to (16) and (17):

$$
\begin{array}{r}
S_{m}(t)=E\left[\exp \left\{-\int_{0}^{t} \theta(u) \lambda(u, \Psi) d u\right\},\right. \\
\lambda_{m}(t)=\theta(t) E[\mu(t, \Psi) \mid T \geq t] .
\end{array}
$$

It is clear that we can formally combine these two models in a single one and obtain the corresponding expectations with respect to two random variables.

There can be different models for $\lambda(t, \Psi)$, the multiplicative one being the simplest:

$$
\lambda(t, \Psi)=\Psi \lambda(t)
$$

where $\lambda(t)$, as usually in the proportional hazards-type models, plays a role of a baseline (reference) rate. Then equation (21) turns into: 


$$
\lambda_{m}(t)=\theta(t) E[\Psi \lambda(t) \mid T \geq t]
$$

Assume that the baseline rate $\lambda(t)$ in equations (22) and (23) is increasing, which models the increasing in time intensity of the doubly stochastic Poisson process $\hat{P}_{t}, t \geq 0$. The observed failure rate $\lambda_{m}(t)$, however, can have a different shape due to the fact that conditional expectations in these formulas are decreasing in time [12]. The following example shows that the resulting mixture failure rate $\lambda_{m}(t)$ can even tend to 0 as $t \rightarrow \infty$.

Example 2. Consider a linearly increasing with age in each realization the rate of the Poisson process of shocks: $\lambda(t, \Psi)=\Psi t$ and assume that $\Psi$ is gamma distributed with parameters $\beta$ and $\vartheta$. Then, in accordance with [12], the failure rate $\lambda_{m}(t)$ defined by equation (23) is

$$
\lambda_{m}(t)=\frac{\beta t}{\vartheta+t^{2}}
$$

This function is equal to zero at $t=0$ and tends to zero as $t \rightarrow \infty$ with a single maximum. at $t=\sqrt{\theta}$. Hence, the mixture of IFR distributions has a decreasing (tending to zero!) failure rate for sufficiently large $t$ and this is rather surprising. Furthermore, the same result asymptotically holds for $\lambda(t)=t^{\alpha}, \alpha>0$.

\section{SHOCKS AS BURN-IN}

We can look at the problem of the failure rate modeling in heterogeneous populations from a different point of view. Now we have an object (a system) with a non-ideal reliability subject to a process of possibly killing shocks. Consider a heterogeneous population of these objects. A manufacturer wants to perform a burn-in procedure, eliminating the weakest items by suitably defined magnitude of a shock. We are interested in comparison of population failure rates before and after the shock. It is natural to suggest that the mixture failure rate after a shock should be smaller than the one before it, otherwise the burn-in of this kind does not make sense. In what follows we define natural conditions which guarantee ordering of failure rates of the described type. 
Let $T \geq 0$ be the time to failure of a system with the Cdf $F(t)$. Let heterogeneity be modeled by the unobserved random variable $\mathrm{Z}$ with support in $[0, \infty)$ (it is more convenient to use this notation for frailty in what follows). Assume that $F(t)$ is indexed by $Z$ :

$$
P(T \leq t \mid Z=z) \equiv P(T \leq t \mid z)=F(t, z)
$$

As the failure rate is a conditional characteristic, similar to previous sections, the mixture failure rate $\lambda_{m}(t)$ should be defined conditionally (see, e.g., [12], [13], [14] to name a few):

$$
\lambda_{m}(t)=\frac{\int_{0}^{\infty} f(t, z) \pi(z) d z}{\int_{0}^{\infty} \bar{F}(t, z) \pi(z) d z}=\int_{0}^{\infty} \lambda(t, z) \pi(z \mid t) d z
$$

where the conditional pdf (on condition that $T \geq t$ ) is:

$$
\pi(z \mid t)=\pi(z) \frac{\bar{F}(t, z)}{\int_{0}^{\infty} \bar{F}(t, z) \pi(z) d z} .
$$

Assume that at time $t=t_{1}$ an instantaneous shock had occurred, which affects the whole population: with the corresponding complementary probabilities, as in Section 1 , it either kills an item, or 'leaves it unchanged'. Without loosing generality, let $t_{1}=0$, otherwise a new initial mixing variable can be easily defined and the corresponding procedure adjusted to this case. It is natural to suppose that the more frail individuals or subpopulations (with larger failure rates) are, the more susceptible they are to failures from a shock.

Let $\pi_{1}(z)$ denote a frailty distribution of a random variable $Z_{1}$ after a shock and let $\lambda_{m s}(t)$ be the corresponding observed (mixture) failure rate after it. Assume:

$$
\pi_{1}(z)=\frac{g(z) \pi(z)}{\int_{0}^{\infty} g(z) \pi(z) d z}
$$

where $g(z)$ is a decreasing function and therefore $\pi_{1}(z) / \pi(z)$ is decreasing. It means that a shock performs a kind of a burn-in operation (Block et al, [15]), and that $Z$ and $Z_{1}$ are ordered in the sense of the likelihood ratio (Ross, [16]):

$$
Z \geq_{L R} Z_{1}
$$


Now we able to formulate the following result, which will be proved in the Appendix:

Theorem Let relation (26), defining a mixing density after a shock at $t=0$, where $g(z)$ is a decreasing function, hold.

Assume that a family of failure rates in a population is ordered in $z$ :

$$
\lambda\left(t, z_{1}\right)<\lambda\left(t, z_{2}\right), \quad z_{1}<z_{2}, \forall z_{1}, z_{2} \in[0, \infty], t \geq 0 .
$$

Then:

$$
\lambda_{m s}(t)<\lambda_{m}(t) ; \forall t \in[0, \infty)
$$

In accordance with inequality (29), the curve $\lambda_{m s}(t)$ lies beneath the curve $\lambda_{m}(t)$ for $t \geq 0$. Therefore, the failure rate after the shock is always smaller than the one without the burn-in of this kind, which, of course, is a natural result for this operation. At $t=0$, for instance:

$$
\lambda_{m}(0)-\lambda_{m s}(0)=\int_{0}^{\infty} \lambda(0, z)\left(\pi(z)-\pi_{1}(z)\right) d z
$$

Ordering (29) seems intuitively evident, but, in fact, it is valid only due to rather stringent conditions of this theorem. It can be shown, for instance, that the replacement of condition (27) by a weaker one, e.g., of stochastic dominance: $Z \geq_{s t} Z_{1}$ will not guarantee ordering this ordering for all $t$. In the latter case it will hold only for sufficiently small $t$.

Ordering (28) is an important assumption, as it, in fact, defines heterogeneity in terms of failure rates of different subpopulations. The simplest and widely used example of the natural ordering of this kind is the proportional hazards model, when $\lambda(t, z)=z \lambda(t)$.

This result can be generalized to the sequence of shocks of the described type at time instants $\left\{t_{i}\right\}, i=1,2, \ldots$ and after every shock the failure rate will be 'lower' than after the previous one.

\section{CONCLUDING REMARKS}

External point influences (shocks) present a common cause of system failures in different applications. The ability to survive a single shock and the intensity of these 
shocks in time eventually define system reliability. There can be different criterions of failure for systems subject to shocks. Only two simplest criterions were considered, but the suggested approach allows for various generalizations.

Heterogeneity is a natural feature in many populations. Results of reliability (safety) analysis for heterogeneous populations can be substantially different from those in a homogeneous case and this should be taken into account. The subjective approach based on considering of an unobserved random variable (frailty) gives an appropriate tool for stochastic modeling of heterogeneity and presents a more flexible way of stochastic description of lifetimes.

Appendix. Proof of the Theorem.

Inequality (28) is a natural ordering in the family of failure rates $\lambda(t, z), z \in[0, \infty)$ and trivially holds for the specific multiplicative model $\lambda(t, z)=z \lambda(t)$.

It can be finally derived that

$$
\begin{gathered}
\operatorname{sign}\left[\lambda_{m s}(t)-\lambda_{m}(t)\right] \\
=\operatorname{sign} \int_{0}^{\infty} \int_{0}^{\infty} \bar{F}(t, u) \bar{F}(t, s)(\lambda(t, u)-\lambda(t, s))\left(\pi_{1}(u) \pi(s)-\pi_{1}(s) \pi(u)\right) d u d s,
\end{gathered}
$$

which is negative due to assumptions of the Theorem. It can be seen, noting that $\operatorname{sign}\left[\lambda_{m s}(t)-\lambda_{m}(t)\right]$ is equal to the sign of

$$
\begin{gathered}
\int_{0}^{\infty} \lambda(t, z) \bar{F}(t, z) \pi_{1}(z) d z \int_{0}^{\infty} \bar{F}(t, z) \pi(z) d z-\int_{0}^{\infty} \lambda(t, z) \bar{F}(t, z) \pi(z) d z \int_{0}^{\infty} \bar{F}(t, z) \pi_{1}(z) d z \\
=\int_{0}^{\infty} \int_{0}^{\infty} \bar{F}(t, u) \bar{F}(t, s)\left[\lambda(t, u) \pi_{1}(u) \pi(s)-\lambda(t, s) \pi_{1}(u) \pi(s)\right] d u d s \\
=\int_{0}^{\infty} \int_{0}^{\infty} \bar{F}(t, u) \bar{F}(t, s)\left[\pi_{1}(u) \pi(s)(\lambda(t, u)-\lambda(t, s))+\pi_{1}(s) \pi(u)(\lambda(t, s)-\lambda(t, u))\right] d u d s \\
=\int_{0}^{\infty} \int_{0}^{\infty} \bar{F}(t, u) \bar{F}(t, s)(\lambda(t, u)-\lambda(t, s))\left(\pi_{1}(u) \pi(s)-\pi_{1}(s) \pi(u)\right) d u d s
\end{gathered}
$$

where we, at first, had transformed the product of integrals into a double integral and then have changed the domain of integration. 


\section{References}

1. Barlow R. and Proschan F. Statistical Theory of Reliability and Life Testing Probability Models, Holt, Rinehart and Winston. 1975..

2. Thompson W.A. Jr. Point process models with applications to safety and reliability, Chapman and Hall, 1988.

3. Aven, T., and Jensen U. Stochastic Models in Reliability. Springer, 1999.

4. Finkelstein M.S. A point-process stochastic model with application to safety at sea. Reliability Engineering and System Safety, 1998, 60, 227-223.

5. Kalashnikov V. Geometric Sums: Bounds for Rare Events with Applications Kluwer Academic Publishers, 1997.

6. Cox D.R. and Isham V. Point processes, Chapman and Hall, London, 1980.

7. Kalbfleish, J.D. and Prentice, R.L. The Statistical Analyses of Failure Time Data, John Wiley \& Sons, 1980.

8. Block H.W, Borges W, Savits T.H. Age dependent minimal repair, J. Appl. Prob. $1985,22,370-386$.

9. Finkelstein, M.S., and Zarudnij V.I. A shock process with a non-cumulative damage. Reliability Engineering and System Safety, 2000, 71, 103-107.

10. Vaupel, J.W., Manton K.G., and Stallard E. The impact of heterogeneity in individual frailty on the dynamics of mortality. Demography, 1979 16, 439-454.

11. Yashin A., and Manton K. Effects of unobserved and partially observed covariate processes on system failure: a review of models and estimation strategies Statistical Science, 1997, 12, 20-34

12. Finkelstein, M.S., and V. Esaulova, V. Modeling a failure rate for the mixture of distribution functions. Probability in Engineering and Informational Sciences, 2001, 15, 383-400.

13. Finkelstein, M.S. Minimal repair in heterogeneous populations. Journal of Applied Probability, 2004, 41, 281-286.

14. Shaked, M and Spizzichino, F. Mixtures and monotonicity of failure rate functions. In: Advances in Reliability (N. Balakrishnan and C.R. Rao-eds), Elsevier: Amsterdam, 2001.

15. Block, H. W., Mi, J., and Savits, T. H.. Burn-in and mixed populations. J. Appl. Prob. 1993, 30, 692-702. 
16. Ross S.M. Stochastic Processes. John Wiley \& Sons, 1996. 